\title{
Synchronization Reveals Topological Scales in Complex Networks
}

\author{
Alex Arenas, ${ }^{1}$ Albert Díaz-Guilera, ${ }^{2}$ and Conrad J. Pérez-Vicente ${ }^{2}$ \\ ${ }^{1}$ Departament d'Enginyeria Informàtica i Matemàtiques, Universitat Rovira i Virgili, 43007 Tarragona, Spain \\ ${ }^{2}$ Departament de Física Fonamental, Universitat de Barcelona, Martí i Franquès 1, 08028 Barcelona, Spain
}

(Received 30 November 2005; published 22 March 2006)

\begin{abstract}
We study the relationship between topological scales and dynamic time scales in complex networks. The analysis is based on the full dynamics towards synchronization of a system of coupled oscillators. In the synchronization process, modular structures corresponding to well-defined communities of nodes emerge in different time scales, ordered in a hierarchical way. The analysis also provides a useful connection between synchronization dynamics, complex networks topology, and spectral graph analysis.
\end{abstract}

DOI: $10.1103 /$ PhysRevLett.96.114102

PACS numbers: 05.45.Xt, 89.75.Fb

The science of complex networks has been a subject of interest for the physics community in recent years [1-3]. Complex networks are found in fields as diverse as the Internet, the World Wide Web, food webs, and biological and social organizations (see [4] and references therein). Although the main characteristics of complex networks have been properly described at the microscale level (node properties) and also at the macroscale level (whole network properties), some of the characteristics of the mesoscale are still elusive. In particular, the community detection problem concerning the determination of mesoscopic structures that have functional, relational, or even social entities is still controversial, starting from the "a priori" definition of what a community is $[5,6]$.

The community detection problem consists of finding a "good" partition of the network in subgraphs that represent communities according to a given definition. However, in many complex networks the organization of nodes is not completely represented by a unique partition, but by a set of nested communities that appear at different topological scales. Let us consider as a naive example the network formed by all human acquaintances. Thus, at some topological scale we can expect to find many communities formed by families and friends and, soon after this scale, the expected partitions into cities will come up; beyond this regions, followed by countries, and, finally, probably continental areas. Here, we aim at giving a method to reveal these different topological scales.

In a completely different scenario, physicists have largely studied the dynamics of complex biological systems, and, in particular, the paradigmatic analysis of large populations of coupled oscillators [7-9]. The emergence of synchronization patterns in these systems has been shown to be closely related to the underlying topology of interactions. In this Letter we show that, for a suitable model, the dynamical process towards synchronization shows different patterns over time intrinsically connected with the hierarchical organization of communities in complex networks. The ubiquity of synchronization phenomena in the real world makes this approach appealing from a physical and biological perspective. Moreover, we will show that the connections with the spectral theory of the Laplacian matrix of a graph spreads the possibilities of the analysis to any complex network.

One of the most successful attempts to understand synchronization phenomena was from Kuramoto [9], who analyzed a model of phase oscillators coupled through the sine of their phase differences. The model is rich enough to display a large variety of synchronization patterns and sufficiently flexible to be adapted to many different contexts [10]. The Kuramoto model consists of a population of $N$ coupled phase oscillators where the phase of the $i$ th unit, denoted by $\theta_{i}(t)$, evolves in time according to the following dynamics

$$
\frac{d \theta_{i}}{d t}=\omega_{i}+\sum_{j} K_{i j} \sin \left(\theta_{j}-\theta_{i}\right) i=1, \ldots, N,
$$

where $\omega_{i}$ stands for its natural frequency and $K_{i j}$ describes the coupling between units. The original model studied by Kuramoto assumed mean-field interactions $K_{i j}=$ $K, \forall i, j$. If the oscillators are identical $\left(\omega_{i}=\omega \forall i\right)$ there is only one attractor of the dynamics: the fully synchronized regime where $\theta_{i}=\theta, \forall i$. Recently, due to the realization that many networks in nature have complex topologies, these studies have been extended to complex networks with local interaction [11-18].

In particular, it has been shown $[19,20]$ that high densely interconnected sets of oscillators (motifs) synchronize more easily that those with sparse connections. This scenario suggests that for a complex network with a nontrivial connectivity pattern, starting from random initial conditions, those highly interconnected units forming local clusters will synchronize first and then, in a sequential process, larger and larger spatial structures will do the same up to the final state where the whole population should have the same phase. We expect this process to occur at different time scales if a clear community structure exists. Thus, the dynamical route towards the global attractor will reveal different topological structures, presumably those which represent communities. Therefore, it is the complete dy- 
namical process what unveils the whole organization at all scales, from the microscale at very early stages up to the macroscale at the end of the time evolution. On the contrary, those systems endowed with a regular topological structure will display a trivial dynamics with a single time scale for synchronization.

To study this phenomena, instead of considering a global observable, we define a local order parameter measuring the average of the correlation between pairs of oscillators

$$
\rho_{i j}(t)=\left\langle\cos \left[\theta_{i}(t)-\theta_{j}(t)\right]\right\rangle
$$

where the brackets stand for the average over initial random phases. The main advantage of this approach is that it allows us to trace the time evolution of pairs of oscillators and therefore to identify compact clusters reminiscent of the existence of communities.

To give evidence of the aforementioned facts, we have analyzed the dynamics towards synchronization - the time evolution of $\rho_{i j}(t)$-in computer-generated graphs with a hierarchical community structure. In [21] the authors proposed models of networks with a well-defined community structure that has been used as a benchmark for different community detection algorithms [6]. Here, we propose a generalization of this model that includes two hierarchical levels of communities. The graphs we generate are as follows: we prescribe, in a set of 256 nodes, 16 compartments that will represent our first community organizational level, and 4 compartments containing each one four different compartments of the above first level, which define the second organizational level of the network. The internal degree of nodes at first level $z_{\mathrm{in}_{1}}$ and the internal degree of nodes at second level $z_{\mathrm{in}_{2}}$ keep an average degree $z_{\mathrm{in}_{1}}+z_{\mathrm{in}_{2}}+z_{\text {out }}=18$. From now on, networks with two hierarchical levels are indicated as $z_{\mathrm{in}_{1}}-z_{\mathrm{in}_{2}}$, e.g., a network with 13-4 means 13 links with the nodes of its first hierarchical level community (more internal), 4 links with the rest of communities that form the second hierarchical level (more external), and 1 link with any community of the rest of the network.

In Fig. 1 we represent $\rho_{i j}(t)$ at the same time $t$ for two slightly different hierarchical networks 13-4 and 15-2. In the two figures we can identify the two levels of the hierarchical distribution of communities. The network 13-4 (left) is very close to a state in which the four large groups are almost synchronized, whereas the network 152 (right) still presents some of the smaller groups of synchronized oscillators, and the larger group starting to synchronize, coherently with their topological structure.

The visualization of the correlation matrix of the system helps in elucidating the topology of the network. To extract the quantitative information it is useful to introduce some threshold $T$ to convert the correlation matrix into a binary matrix, that will be used to determine the borders between different groups. We define a dynamic connectivity matrix
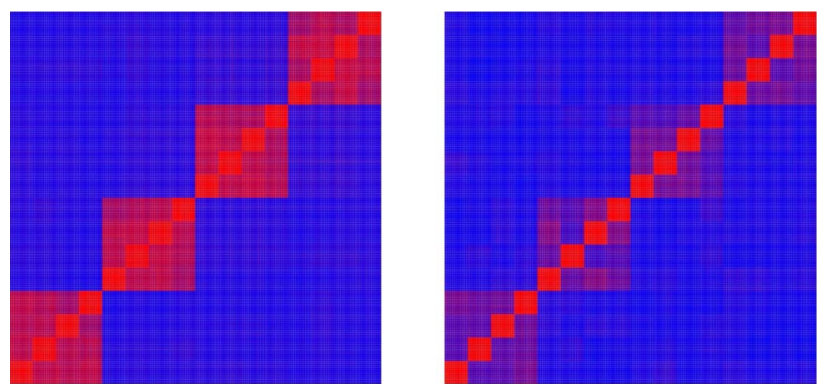

FIG. 1 (color online). Average of the correlation between pairs of oscillators. The structure networks are 13-4 (left) and 15-2(right). See text for a description of the networks. The colors are a gradation between blue (0) and red (1).

$$
\mathcal{D}_{t}(T)_{i j}=\left\{\begin{array}{ll}
1 & \text { if } \rho_{i j}(t)>T \\
0 & \text { if } \rho_{i j}(t)<T
\end{array},\right.
$$

that depends on both the underlying topology and the collective dynamics. For a fixed time $t$, by moving the threshold $T$, we obtain different representations of $\mathcal{D}_{t}(T)$ that inform us about the structure of the dynamic correlations. When the threshold is large enough, the representation of $\mathcal{D}_{t}(T)$ becomes a set of disconnected clumps or communities. Decreasing $T$ a hierarchical structure of communities is devised. Note that since the function $\rho_{i j}(t)$ is continuous and monotonic (because the existence of a unique attractor of the dynamics), we can redefine $\mathcal{D}_{T}(t)$, i.e., fixing the threshold and evolving in time. We obtain the same information about the structure of the dynamic connectivity matrix at different time scales. Let us show that these time scales unravel the topological structure of the connectivity matrix at different topological scales.

From the eigenvalue spectrum of $\mathcal{D}_{T}(t), S\left[\mathcal{D}_{T}(t)\right]$, one can extract the number of disconnected components of the system as the number of null eigenvalues. The evolution of $S\left[D_{T}(t)\right]$ traces the hierarchy of communities as follows: at short times, all units are uncorrelated and then we have $N$ disconnected sets, $N$ being the number of nodes in the network; as time goes on, nodes become synchronized in groups according to their topological structure. In Fig. 2 (top) we plot, for the two networks analyzed in Fig. 1, the number of disconnected components as a function of time, for a fixed threshold $T$ [22]. We can observe the relative stability of the two partitions for the two networks, corresponding to the two prescribed hierarchical levels. For the 13-4 network the synchronization of the 4 groups of 64 nodes each is much more stable than the 16 groups of 16 nodes, i.e., the community structure at the second hierarchical level is stronger, whereas the opposite can be inferred for network 15-2.

Another interesting link between dynamics and topology can be highlighted from the analysis of the whole spectrum of the Laplacian matrix of the network graph $\mathcal{L}$ [23]. The Laplacian matrix is defined as $\mathcal{L}_{i j}=k_{i} \delta_{i j}-a_{i j}$, 


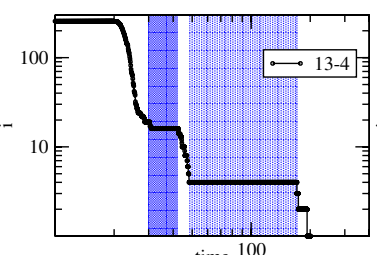

time 100
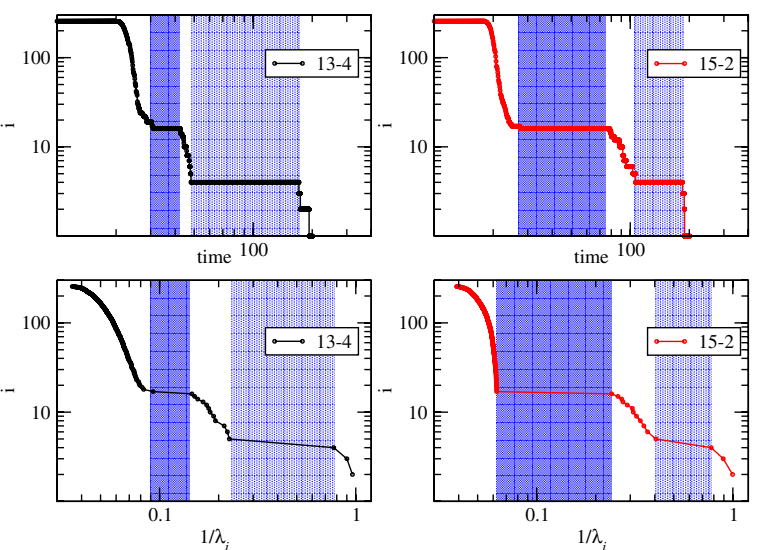

FIG. 2 (color online). Top: Number of disconnected synchronized components (equivalent to number of null eigenvalues of $\left.S\left[\mathcal{D}_{T}(t)\right]\right)$ as a function of time for the two networks of Fig. 1 at $T=0.99$. Bottom: Rank index $i$ (see text) versus the inverse of the corresponding eigenvalues of the Laplacian matrix $\mathcal{L}$. The shadow regions indicate the stability plateaus for 16 (dark) and 4 (light) communities. The same representation is used for the plateaus in the eigenvalue spectrum corresponding to indices 16 and 4.

where $k_{i}$ is the degree of node $i, \delta_{i j}$ is the Kronecker delta, and $a_{i j}$ is the element of the adjacency matrix ( 1 if nodes $i$ and $j$ are connected and 0 otherwise). The spectral information of the Laplacian matrix has been used to understand the structure of complex networks [24], and, in particular, to detect the community structure $[25,26]$. Recent studies have also focused on the spectral information of the Laplacian matrix and the synchronization dynamics [1118]. The common approach is to take advantage of the master stability equation [27] to determine the relation between the relative stability of the synchronized state (via the ratio $\lambda_{N} / \lambda_{2}$ ) and the heterogeneity of the topology, although sometimes some language abuse appears and authors talk about better or worse synchonizability instead of stability of the synchronized state. Our approach differs from these works in the following: we are interested in the transient towards synchronization because it is this whole process which will reveal the topological structure at different scales. For this reason our analysis focuses on the whole eigenvalue spectrum of the Laplacian matrix $S(\mathcal{L})$.

To characterize this spectrum, we rank the eigenvalues of $\mathcal{L}$ using an index $i$ in ascending order $0=\lambda_{1} \leq \lambda_{2} \leq$ $\ldots \lambda_{i} \ldots \leq \lambda_{N}$. The structure of this sequence brings to light many aspects of the topological structure: (i) the number of null eigenvalues gives trivially the number of disconnected components, (ii) the gaps between consecutive eigenvalues tell us about the relative differences of time scales, and (iii) large eigenvalues in the last part of the series stand for the existence of hubs in the network (we will turn to these points later). In Fig. 2 (bottom) we have plotted the eigenvalues of the Laplacian matrix for the 13-4 and 15-2 structures. We observe three groups of eigenval- ues separated by gaps. Each gap separates a community either of 256, 16, or 4 group's elements or the whole population. Notice that for the 13-4 graph the plateau of 16 communities is shorter than the plateau for 4 communities and the contrary is true for the 15-2 case, indicating that the 16 clusters community is less well defined in the former case. Indeed, the ratio between the eigenvalues is a good quantitative measure of the stability of the structure (which is measured in terms of modularity in other studies [6]) and is related to the length of the plateaus observed in Fig. 2 (top).

We visualize the formation of the connected groups of synchronized oscillators in time by constructing a dendogram in which we draw lines between groups of oscillators when they merge. Applying this technique to the above defined networks we can see two different topological scales disclosed by synchronization and the relative stability of them. The networks investigated so far are homogeneous in degree. At this point we ask about the effect when inhomogeneities in degree are considered. We have applied this procedure to the network structure proposed by Ravasz and Barabasi [28] with a hierarchical structure in two levels and a scale-free degree distribution. As can be seen from the dendogram depicted in Fig. 3, the communities synchronize at different times, depending on their role in the hierarchy, and it also shows the remarkable effect of hubs in the synchronization process.

Finally we would like to shed some light about the intriguing relationship between the eigenvalues of the Laplacian and the dynamic structures that emerge towards synchronization. To understand this correspondence let us analyze the linearized dynamics of the Kuramoto model (i.e., the dynamics close to the attractor of synchronization) in terms of the Laplacian matrix,
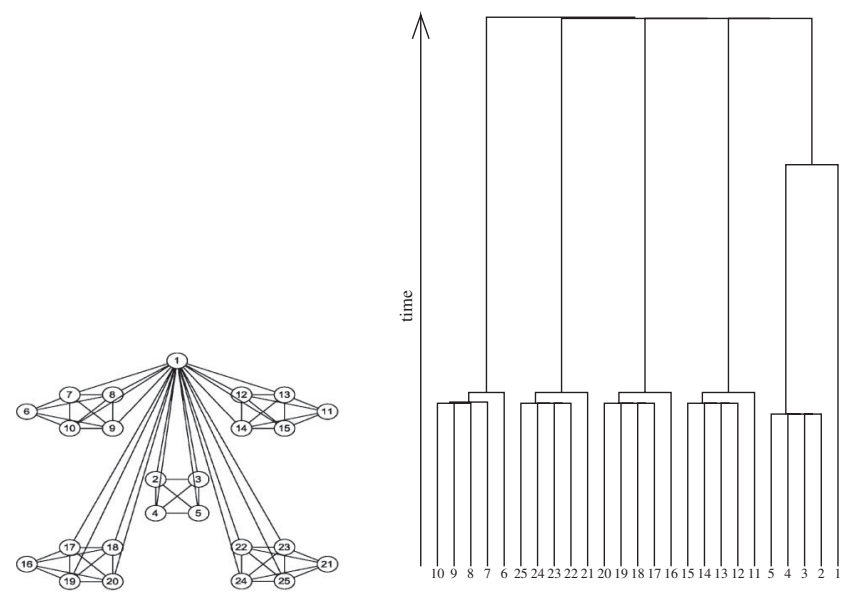

FIG. 3. Left: Ravasz-Barabasi network of 25 labeled nodes with two hierarchical levels. Right: Time evolution of the synchronization process between labeled oscillators. The length of the dendogram branches indicate the relative stability of the different structures. 


$$
\frac{d \theta_{i}}{d t}=-k \sum_{j} L_{i j} \theta_{j} i=1, \ldots, N,
$$

whose solution in terms of the normal modes $\varphi_{i}(t)$ reads

$$
\varphi_{i}(t)=\sum_{j} B_{i j} \theta_{j}=\varphi_{i}(0) e^{-\lambda_{i} t} i=1, \ldots, N
$$

where $\lambda_{i}$ are the eigenvalues of the Laplacian matrix, and $B$ is the eigenvectors matrix.

This set of equations has to be satisfied at any time $t$. If we rank the system of equations in descending order of the eigenvalues (i.e., starting from $\lambda_{N}$ ), the right-hand side system of Eq. (5) will approach zero in a hierarchical way. This fact is equivalent in the dynamics to group oscillators surpassing the synchronization threshold forming communities. The gaps in the spectrum $S(\mathcal{L})$ clearly represent different time scales between modes revealing different topological scales. The collective modes, the solution of the system represented by Eq. (5), denote two types of behaviors. Some modes provide information about reorganization of the phases in the whole network, while the others inform us about synchronization between pairs or groups of oscillators. The presence of hubs in the topology gives rise to large eigenvalues that decay very fast and are related to the first type of modes, those representing "synchronization" between the hub and the topological average of the phases of rest of oscillators. The rest of the modes relate oscillators that have similar projections on the corresponding eigenvectors, thus giving rise to communities at a given topological scale. Indeed, this fact supports the success of the identification of communities using spectral analysis [25].

Summarizing, we have analyzed the synchronization dynamics in complex networks and show how this process unravels its different topological scales. We have also reported a connection between the spectral information of the Laplacian matrix and the hierarchical process of emergence of communities at different time scales.

We thank M. A. Muñoz, Y. Moreno, and R. Guimerà for helpful comments. This work has been supported by DGES of the Spanish Government Grant No. BFM-2003-08258 and EC-FET Open Project No. IST-2001-33555.

Note added in proof.-M. Sales, R. Guimerà, and L. A. N. Amaral have a paper about a closely related subject: the determination of community hierarchies in complex networks. We are aware of this work by personal communication although the paper has not been publicly available.
[1] S. Strogatz, Nature (London) 410, 268 (2001).

[2] R. Albert and A.-L. Barabasi, Rev. Mod. Phys. 74, 47 (2002).

[3] S. Boccaletti, V. Latora, Y. Moreno, M. Chavez, and D.-U. Hwang, Phys. Rep. 424, 175 (2006).

[4] M. Buchanan, Nexus (Norton, NewYork, 2002).

[5] M. E. J. Newman, Eur. Phys. J. B 38, 321 (2004).

[6] L. Danon, A. Diaz-Guilera, J. Duch, and A. Arenas, J. Stat. Mech. (2005) P09008 .

[7] A.T. Winfree, The Geometry of Biological Time (Springer-Verlag, Berlin 2001), 2nd ed.

[8] S. H. Strogatz, Sync: The Emerging Science of Spontaneous Order (Hyperion, New York, 2003).

[9] Y. Kuramoto, Chemical Oscillations, Waves, and Turbulence (Dover, Mineola, NY, 2003), 2nd ed.

[10] J. A. Acebron, L. L. Bonilla, C. J. Perez Vicente, F. Ritort, and R. Spigler, Rev. Mod. Phys. 77, 137 (2005).

[11] M. Barahona and L. M. Pecora, Phys. Rev. Lett. 89, 054101 (2002).

[12] T. Nishikawa, A.E. Motter, Y.-C. Lai, and F. C. Hoppensteadt, Phys. Rev. Lett. 91, 014101 (2003).

[13] Y. Moreno and A.F. Pacheco, Europhys. Lett. 68, 603 (2004).

[14] H. Hong, B. J. Kim, M. Y. Choi, and H. Park, Phys. Rev. E 69, 067105 (2004).

[15] A.E. Motter, C. Zhou, and J. Kurths, Phys. Rev. E 71, 016116 (2005).

[16] D.-S. Lee, Phys. Rev. E 72, 026208 (2005).

[17] L. Donetti, P. I. Hurtado, and M. A. Muñoz, Phys. Rev. Lett. 95, 188701 (2005).

[18] M. Chavez, D.-U. Hwang, A. Amann, H. G. E. Hentschel, and S. Boccaletti, Phys. Rev. Lett. 94, 218701 (2005).

[19] Y. Moreno, M. Vazquez-Prada, and A. F. Pacheco, Physica A (Amsterdam) 343, 279 (2004).

[20] E. Oh, K. Rho, H. Hong, and B. Kahng, Phys. Rev. E 72, 047101 (2005).

[21] M.E. J. Newman and M. Girvan, Phys. Rev. E 69, 026113 (2004).

[22] We prescribe the value of the threshold $T$ to 0.999 . Other choices do not alter the results, since it only modifies the relative time scales.

[23] N.L. Biggs, Algebraic Graph Theory (Cambridge University Press, Cambridge, England, 1974).

[24] I. J. Farkas, I. Derenyi, A.-L. Barabasi, and T. Vicsek, Phys. Rev. E 64, 026704 (2001).

[25] L. Donetti and M. A. Muñoz, J. Stat. Mech. (2004)P10012.

[26] A. Capocci, V.D.P. Servedio, G. Caldarelli, and F. Colaiori, Physica A (Amsterdam) 352, 669 (2005).

[27] L. M. Pecora and T. L. Carroll, Phys. Rev. Lett. 80, 2109 (1998).

[28] E. Ravasz and A.-L. Barabasi, Phys. Rev. E 67, 026112 (2003). 